\title{
Childhood Pneumonia Research in India: Current and Future Perspectives
}

\author{
JOSEPH L. MATHEW \\ Professor (Pediatric Pulmonology), Advanced Pediatric Centre, PGIMER Chandigarh. \\ dr.joseph.l.mathew@gmail.com
}

$\mathrm{C}$ hildhood community acquired pneumonia (CAP) continues to remain a problem of great clinical and public health significance. Its impact on individual children, the community, and the healthcare system, is probably unrivalled by any other childhood disease. For several decades, India has been faithfully following the formal and informal guidance provided by international agencies including the World Health Organization (WHO), reputed global funding agencies, and prestigious universities/institutions/organizations promoting research. Local data has generally been limited in terms of quality as well as quantity. In that context, it is laudable that Indian Pediatrics has focused this issue on research and topics related to childhood pneumonia. This has been possible largely through the support of the International Clinical Epidemiology Network (INCLEN) based in New Delhi, which initiated and executed a nationallevel research program on childhood pneumonia.

This issue of the Journal carries seven publications flowing from this initiative [1-7], besides two other independent research studies [8,9]. An external evaluation of the entire initiative, by a team of globally renowned researchers, is also presented in this issue [10]. Some of these studies have provided confirmation of known results, but in the Indian context. Most have used standard methods and/ or tools to re-explore issues that are generally accepted or expected.

This begets the question of what has been achieved through the excellent effort initiated by INCLEN. First, almost all the studies [1-7] highlight considerable attention to methodological rigor, including clearly articulated research questions, appropriate study designs, a priori sample size calculations, multi-centric nature of some studies, fairly large sample sizes, and efforts to limit some sources of bias. This clearly reflects the role of INCLEN, in terms of design, execution, mentorship, and oversight of the research studies. The second, and perhaps greater, gain is the establishment of a network of institutions and researchers across the length and breadth of the country (except Eastern region), with an interest in childhood pneumonia. This bodes well for the reasons highlighted subsequently. Third, previous experience with INCLEN supported research studies, shows that this prestigious agency continues working beyond the completion of the research studies, towards widespread dissemination of the data, advocacy with policy-makers and other key decision-makers, and translating the evidence into actionable plans. Thus, INCLEN has acted much more, and much better than a traditional "funding agency". Its efforts will provide great thrust not only to research on childhood CAP, but its practical translation to policy and practice.

Although the external evaluation [10] identified that the program met its objectives, some areas of concern remain. The program itself was funded by the Bill and Melinda Gates Foundation, hence the selection of research project proposals was based on alignment to the priorities of the Foundation, not necessarily our country. This raises the issue of what our country's priorities should be, in the area of childhood CAP research. To my mind, the following questions provide a broad outline:

- What is the microbial etiology of pneumonia in individual children in the community (and not hospitalized children alone)?

- How to distinguish bacterial from non-bacterial etiology in individual children with CAP, at presentation, and at the point-of-care?

- Which features in individual children, at presentation, and at the point-of-care, are associated with clinical deterioration and/or adverse outcome?

- What tools and support can be provided to (community) healthcare workers for appropriate (i.e., not merely empiric) management of children at the point-of-care?

- What is the impact of environmental (external and internal) factors in the initiation, progression, and final outcome of children with pneumonia?

- Which internal host factors influence the onset, course, and outcome, of pneumonia (beyond the wellrecognized macro-level factors such as nutritional 
status, breastfeeding, vaccination, exposure to smoke, etc.)?

- What is the prevalence, pattern and outcome of CAP in infants younger than 2 month and older than 5 year?

- Given that measles pathophysiology starts with lower respiratory tract infection, what strategies could/should be used to rapidly eliminate measles in the country?

- What is the burden of RSV infection in childhood pneumonia (distinct from bronchiolitis), and can we rapidly generate evidence to manage it appropriately?

These questions reflect three important facts. First, hospital-based studies may be inappropriate to address the questions related to disease affecting children in the community. Second, the focus of research should shift from cohorts to individual children. Third, research needs to focus on host and environmental issues rather than microbes alone. Additionally, the first two questions are critical to evolve appropriate treatment and prophylaxis decisions, rather than indiscriminately following the approach handeddown by external agencies.

Current global research has already shifted focus from Pneumococcus to RSV [11-15], setting the ground for the anticipated roll-out of vaccines and/or other prophylaxis strategies. Therefore, the last question highlighted above needs urgent answers, lest India be caught in the unenviable position of lacking local data, but facing pressure to initiate expensive prophylaxis programs. Previous experience of our country with several other vaccines suggests that this scenario is very likely to recur.

Although the program did not address any of these crucial issues, it still carries great potential, provided $(i)$ the network of institutions and individual researchers can be preserved despite the completion of the studies; (ii) the sites in the tertiary-care institutions can begin engaging with the local community for future community-based research; (iii) funding can be attracted from local and international agencies; $(i v)$ additional institutions along with their satellites can be added; and $(v)$ the same level of mentorship, oversight, and monitoring can be maintained. I believe that this will not only make our country self-reliant in evidencebased policy and practice decision-making for childhood pneumonia, but pave the path for similar self-reliance in other areas of child health also.

Funding: None. Competing interest: None stated.

\section{REFERENCES}

1. Bhat JI, Charoo BA, Mukherjee A, et al. Risk of Hospitalization in Under-five Children with Community - A
Multicentric Prospective Cohort Study. Indian Pediatr 2021; 58:1019-23.

2. Das RR, Ratageri VH, Lodha R, ATU Group. Role of Clinical Criteria and Oxygen Saturation Monitoring in Diagnosis of Childhood Pneumonia in Children Aged 2 to 59 Months. Indian Pediatr 2021; 58:1024-9.

3. Kumar S, Mohanraj R, Dhingra B, Suresh S. Optimizing CareSeeking for Childhood Pneumonia: A Public Health Perspective. Indian Pediatr 2021; 58:1030-5.

4. Goyal JP, Kumar P, Mukherjee A, et al. Risk Factors for the Development of Pneumonia and Severe Pneumonia in Children. Indian Pediatr 2021; 58:1036-9.

5. Kumar CS, Subramanian S, Murki S, et al. Predictors of Mortality in Neonatal Pneumonia: An INCLEN Childhood Pneumonia Study. Indian Pediatr 2021; 58:1040-5.

6. Gothankar J, Pore P, Dhumale G, et al. Behavior Change Communication on the Incidence of Pneumoniain Under Five Children: A Cluster Randomized Controlled Trial. Indian Pediatr. 2021; 58:1046-51.

7. Nair NS, Lewis LE, Dhyani VS. Factors Associated with Neonatal Pneumonia and its Mortality in India: A Systematic Review and Meta-Analysis. Indian Pediatr 2021; 58:1059-66.

8. Pillai K, Sartho ER, Lakshmi TP, Parvathy VK. Diagnosis and Assessment of Severity of Pediatric Pneumonia Using the Respiratory Index of Severity (RISC) Scoring System. Indian Pediatr 2021; 58:1052-5.

9. Huong PLT, Hien PT, Lan NTP, Tuan DM, Anh DD, Binh TQ. Clinical Patterns and Risk Factors for Pneumonia Caused by atypical bacteria in Vietnamese children. Indian Pediatr. 2021;58:1056-8.

10. Cherian T, Gupta P, Thomas K. An external evaluation on the INCLEN research program to emphasize the public health significance. Indian Pediatr. 2021;58:1074-6.

11. Shi T, McAllister DA, O’Brien KL, et al. Global, regional, and national disease burden estimates of acute lower respiratory infections due to respiratory syncytial virus in young children in 2015: a systematic review and modelling study. Lancet. 2017;390:946-58.

12. Baral R, Higgins D, Regan K, Pecenka C. Impact and costeffectiveness of potential interventions against infant respiratory syncytial virus (RSV) in 131 low-income and middle-income countries using a static cohort model. BMJ Open. 2021;11:e046563.

13. Pneumonia Etiology Research for Child Health (PERCH) Study Group. Causes of severe pneumonia requiring hospital admission in children without HIV infection from Africa and Asia: the PERCH multi-country case-control study. Lancet. 2019;394:757-79.

14. Zhang S, Akmar LZ, Bailey F, et al. Cost of respiratory syncytial virus-associated acute lower respiratory infection management in young children at the regional and global level: a systematic review and meta-analysis. J Infect Dis. 2020;222(Suppl 7):S680-87.

15. No authors listed. The global respiratory syncytial virus burden.Accessed: 24 October, 2021.Available from: https:// path.azureedge.net/media/documents/CVIA_RSV_disease_ burden_fs.pdf 